\title{
Growth properties of composite analytic functions of several complex variables in unit polydisc under the treatment of slowly changing functions
}

\author{
Sanjib Kumar Datta ${ }^{1}$, Tanmay Biswas ${ }^{2}$, Ahsanul Hoque ${ }^{3}$ \\ ${ }^{1}$ Department of Mathematics, University of Kalyani, P.O.-Kalyani, Dist.-Nadia, PIN- 741235, West Bengal, India. \\ ${ }^{2}$ Rajbari, Rabindrapalli, R. N. Tagore Road, P.O.-Krishnagar, Dist.-Nadia, PIN-741101, West Bengal, India. \\ ${ }^{3}$ Department of Mathematics, University of Kalyani, P.O.-Kalyani, Dist.-Nadia, PIN- 741235, West Bengal, India.
}

Received: 18 June 2016, Accepted: 14 August 2016

Published online: 27 April 2017.

Abstract: In the paper we establish some new results depending on the comparative growth properties of composite entire or meromorphic functions using relative $L^{*}$-order and relative $L^{*}$-lower order as compared to their corresponding left and right factors.

Keywords: Entire function, meromorphic function, composition, growth, relative $L^{*}$-order, relative $L^{*}$-lower order, slowly changing function.

\section{Introduction, definitions and notations}

A function $f$, analytic in the unit $\operatorname{disc} U=\{z:|z|<1\}$, is said to be of finite Nevanlinna order [4] if there exist a number $\mu$ such that Nevanlinna characteristic function

$$
T_{f}(r)=\frac{1}{2 \pi} \int_{0}^{2 \pi} \log ^{+}\left|f\left(r e^{i \theta}\right)\right| d \theta
$$

satisfies $T_{f}(r)<(1-r)^{-\mu}$ for all $r$ in $0<r_{0}(\mu)<r<1$. The greatest lower bound of all such numbers $\mu$ is called the Nevanlinna order of $f$. Thus the Nevanlinna order $\rho_{f}$ of $f$ is given by

$$
\rho_{f}=\limsup _{r \rightarrow 1} \frac{\log T_{f}(r)}{-\log (1-r)} .
$$

Similarly, the Nevanlinna lower order $\lambda_{f}$ of $f$ is given by

$$
\lambda_{f}=\liminf _{r \rightarrow 1} \frac{\log T_{f}(r)}{-\log (1-r)} .
$$

Somasundaram and Thamizharasi [6] introduced the notions of $L$-order ( $L$-lower order ) for entire functions where $L \equiv$ $L(r)$ is a positive continuous function increasing slowly i.e., $L(a r) \sim L(r)$ as $r \rightarrow \infty$ for every positive constant ' $a$ '. In the line of Somasundaram and Thamizharasi [6], one may introduce the notion of Nevanlinna $L$-order for an analytic function 
$f$ in the unit disc $U=\{z:|z|<1\}$ where $L \equiv L\left(\frac{1}{1-r}\right)$ is a positive continuous function in the unit disc $U$ increasing slowly i.e., $L\left(\frac{a}{1-r}\right) \sim L\left(\frac{1}{1-r}\right)$ as $r \rightarrow 1$, for every positive constant $a$, in the following manner.

Definition 1. If $f$ be analytic in $U$, then the Nevanlinna L-order $\rho_{f}^{L}$ of $f$ is defined as

$$
\rho_{f}^{L}=\inf \left\{\mu>0: T_{f}(r)<\left[\frac{L\left(\frac{1}{1-r}\right)}{(1-r)}\right]^{\mu} \text { for all } 0<r_{0}(\mu)<r<1\right\}
$$

Similarly one may define $\lambda_{f}^{L}$, the Nevanlinna $L$-lower order of $f$ in the following way.

$$
\lambda_{f}=\liminf _{r \rightarrow 1} \frac{\log T_{f}(r)}{\log \left(\frac{L\left(\frac{1}{1-r}\right)}{(1-r)}\right)} .
$$

The more generalised concept of the Nevanlinna $L$-order and the Nevanlinna $L$-lower order an analytic function $f$ in the unit disc $U$ are the Nevanlinna $L^{*}$-order and the Nevanlinna $L^{*}$-lower order. Their definitions are as follows.

Definition 2. [2] The Nevanlinna $L^{*}$-order $\rho_{f}^{L^{*}}$ and Nevanlinna $L^{*}$-lower order $\lambda_{f}^{L^{*}}$ of an analytic function $f$ in the unit disc $U$ are defined as

$$
\begin{aligned}
& \rho_{f}^{L^{*}}=\limsup _{r \rightarrow 1} \frac{\log T_{f}(r)}{\log \left(\frac{\exp \left\{L\left(\frac{1}{1-r}\right)\right\}}{(1-r)}\right)} \text { and } \\
& \lambda_{f}^{L^{*}}=\liminf _{r \rightarrow 1} \frac{\log T_{f}(r)}{\log \left(\frac{\exp \left\{L\left(\frac{1}{1-r}\right)\right\}}{(1-r)}\right)} \text { respectively. }
\end{aligned}
$$

Extending the notion of single variables to several variables, let $f\left(z_{1}, z_{2}, \cdots, z_{n}\right)$ be a non-constant analytic function of $n$ complex variables $z_{1}, z_{2}, \cdots, z_{n-1}$ and $z_{n}$ in the unit polydisc.

$$
U=\left\{\left(z_{1}, z_{2}, \cdots, z_{n}\right):\left|z_{j}\right| \leq 1, j=1,2, \cdots, n ; r_{1}>0, r_{2}>0, \cdots, r_{n}>0\right\}
$$

Now in the line of Nevanlinna $L^{*}$-order and Nevanlinna $L^{*}$-lower order, in this paper we introduce the Nevanlinna $n$ variables based $L^{*}$-order and the Nevanlinna $n$ variables based $L^{*}$-lower order for functions of $n$ complex variables analytic in a unit polydisc as follows.

$$
{ }_{v_{n}} \rho_{f}^{L^{*}}=\limsup _{r_{1}, r_{2}, \cdots, r_{n} \rightarrow 1} \frac{\log T_{f}\left(r_{1}, r_{2}, \cdots, r_{n}\right)}{\log \left[\frac{\exp \left\{L\left(\frac{1}{1-r_{1}}, \frac{1}{1-r_{2}}, \cdots, \frac{1}{1-r_{n}}\right)\right\}}{\left(1-r_{1}\right)\left(1-r_{2}\right) \cdots\left(1-r_{n}\right)}\right]}
$$

and

$$
{ }_{v_{n}} \lambda_{f}^{L^{*}}=\liminf _{r_{1}, r_{2}, \cdots, r_{n} \rightarrow 1} \frac{\log T_{f}\left(r_{1}, r_{2}, \cdots, r_{n}\right)}{\log \left[\frac{\exp \left\{L\left(\frac{1}{1-r_{1}}, \frac{1}{1-r_{2}}, \cdots, \frac{1}{1-r_{n}}\right)\right\}}{\left(1-r_{1}\right)\left(1-r_{2}\right) \cdots\left(1-r_{n}\right)}\right]}
$$

where $L \equiv L\left(\frac{1}{1-r_{1}}, \frac{1}{1-r_{2}}, \cdots, \frac{1}{1-r_{n}}\right)$ is a positive continuous function in the unit polydisc $U$ increasing slowly i.e., $L\left(\frac{a}{1-r_{1}}, \frac{a}{1-r_{2}}, \cdots, \frac{a}{1-r_{n}}\right) \sim L\left(\frac{1}{1-r_{1}}, \frac{1}{1-r_{2}}, \cdots, \frac{1}{1-r_{n}}\right)$ as $r \rightarrow 1$, for every positive constant ' $a$ '. In this paper we study some growth properties of Nevanlinna's Characteristic function relating to the composition of two analytic functions in the unit polydisc on the basis of Nevanlinna $n$ variables based $L^{*}$-order and Nevanlinna $n$ variables based $L^{*}$-lower order as compared to the growth of their corresponding left and right factors.. We do not explain the standard definitions and notations in the theory of entire functions as those are available in [1], [3] and [5]. 


\section{Theorems}

In this section we present the main results of the paper.

Theorem 1. Let $f$ and $g$ be any two non-constant analytic functions of $n$ complex variables in the unit polydisc $U$ such that $0<{ }_{v_{n}} \lambda_{f \circ g}^{L^{*}} \leq{ }_{v_{n}} \rho_{f \circ g}^{L^{*}}<\infty$ and $0<{ }_{v_{n}} \lambda_{f}^{L^{*}} \leq{ }_{v_{n}} \rho_{f}^{L^{*}}<\infty$. If $L\left(\frac{1}{1-r_{1}}, \frac{1}{1-r_{2}}, \cdots, \frac{1}{1-r_{n}}\right)=o\left\{\log T_{f}\left(r_{1}, r_{2}, \cdots, r_{n}\right)\right\}$ as $r_{1}, r_{2}, \cdots, r_{n} \rightarrow 1$ then

$$
\begin{aligned}
& \frac{v_{n} \lambda_{f \circ g}^{L^{*}}}{v_{n} \rho_{f}^{L^{*}}} \leq \liminf _{r_{1}, r_{2}, \cdots, r_{n} \rightarrow 1} \frac{\log T_{f \circ g}\left(r_{1}, r_{2}, \cdots, r_{n}\right)}{\log T_{f}\left(r_{1}, r_{2}, \cdots, r_{n}\right)+L\left(\frac{1}{1-r_{1}}, \frac{1}{1-r_{2}}, \cdots, \frac{1}{1-r_{n}}\right)} \leq \frac{v_{n} \lambda_{f \circ g}^{L^{*}}}{v_{n} \lambda_{f}^{L^{*}}} \\
& \leq \limsup _{r_{1}, r_{2}, \cdots, r_{n} \rightarrow 1} \frac{\log T_{f \circ g}\left(r_{1}, r_{2}, \cdots, r_{n}\right)}{\log T_{f}\left(r_{1}, r_{2}, \cdots, r_{n}\right)+L\left(\frac{1}{1-r_{1}}, \frac{1}{1-r_{2}}, \cdots, \frac{1}{1-r_{n}}\right)} \leq \frac{{ }_{v_{n}} \rho_{f \circ g}^{L^{*}}}{{ }_{v_{n}} \lambda_{f}^{L^{*}}} .
\end{aligned}
$$

Proof. From the definition of Nevanlinna $n$ variables based $L^{*}$-order and Nevanlinna $n$ variables based $L^{*}$-lower order of analytic functions in the unit polydisc $U$, we have for arbitrary positive $\varepsilon$ and for all sufficiently large values of $\left(\frac{1}{1-r_{1}}\right),\left(\frac{1}{1-r_{2}}\right), \cdots$, and $\left(\frac{1}{1-r_{n}}\right)$ that

$\log T_{f \circ g}\left(r_{1}, r_{2}, \cdots, r_{n}\right) \geq\left({ }_{v_{n}} \lambda_{f \circ g}^{L^{*}}-\varepsilon\right) \log \left[\frac{\exp \left\{L\left(\frac{1}{1-r_{1}}, \frac{1}{1-r_{2}}, \cdots, \frac{1}{1-r_{n}}\right)\right\}}{\left(1-r_{1}\right)\left(1-r_{2}\right) \cdots\left(1-r_{n}\right)}\right]$,

$\log T_{f \circ g}\left(r_{1}, r_{2}, \cdots, r_{n}\right) \geq\left({ }_{{ }_{n}} \lambda_{f \circ g}^{[m] L^{*}}-\varepsilon\right)\left[\log \left(\frac{1}{1-r_{1}}\right)+\log \left(\frac{1}{1-r_{2}}\right), \ldots,\left(\frac{1}{1-r_{n}}\right)+L\left(\frac{1}{1-r_{1}}, \frac{1}{1-r_{2}}, \cdots, \frac{1}{1-r_{n}}\right)\right]$

and

$$
\begin{aligned}
& \log T_{f}\left(r_{1}, r_{2}, \cdots, r_{n}\right) \leq\left({ }_{n} \rho_{f}^{L^{*}}+\varepsilon\right) \log \left[\frac{\exp \left\{L\left(\frac{1}{1-r_{1}}, \frac{1}{1-r_{2}}, \cdots, \frac{1}{1-r_{n}}\right)\right\}}{\left(1-r_{1}\right)\left(1-r_{2}\right) \cdots\left(1-r_{n}\right)}\right] \\
& \log T_{f}\left(r_{1}, r_{2}, \cdots, r_{n}\right) \leq\left({ }_{n} \rho_{f}^{L^{*}}+\varepsilon\right)\left[\log \left(\frac{1}{1-r_{1}}\right)+\log \left(\frac{1}{1-r_{2}}\right),\left(\frac{1}{1-r_{n}}\right)+L\left(\frac{1}{1-r_{1}}, \ldots, \frac{1}{1-r_{2}}, \cdots, \frac{1}{1-r_{n}}\right)\right] . \\
& \frac{\log T_{f}\left(r_{1}, r_{2}, \cdots, r_{n}\right)}{\left.{ }_{v_{n}} \rho_{f}^{L^{*}}+\varepsilon\right)} \leq \log \left(\frac{1}{1-r_{1}}\right)+\log \left(\frac{1}{1-r_{2}}\right), \cdots, \log \left(\frac{1}{1-r_{n}}\right)+L\left(\frac{1}{1-r_{1}}, \frac{1}{1-r_{2}}, \cdots, \frac{1}{1-r_{n}}\right) .
\end{aligned}
$$

Now from (1) and (2), it follows for all sufficiently large values of $\left(\frac{1}{1-r_{1}}\right),\left(\frac{1}{1-r_{2}}\right), \cdots$ and $\left(\frac{1}{1-r_{n}}\right)$ that

$$
\log T_{f \circ g}\left(r_{1}, r_{2}, \cdots, r_{n}\right) \geq \frac{\left({ }_{v_{n}} \lambda_{f \circ g}^{L^{*}}-\varepsilon\right)}{\left({ }_{n} \rho_{f}^{L^{*}}+\varepsilon\right)} \log T_{f}\left(r_{1}, r_{2}, \cdots, r_{n}\right)
$$

that is,

$$
\frac{\log T_{f \circ g}\left(r_{1}, r_{2}, \cdots, r_{n}\right)}{\log T_{f}\left(r_{1}, r_{2}, \cdots, r_{n}\right)+L\left(\frac{1}{1-r_{1}}, \frac{1}{1-r_{2}}, \cdots, \frac{1}{1-r_{n}}\right)} \geq \frac{\left(v_{n} \lambda_{f \circ g}^{L^{*}}-\varepsilon\right)}{\left(v_{n} \rho_{f}^{L^{*}}+\varepsilon\right)} \cdot \frac{\log T_{f}\left(r_{1}, r_{2}, \cdots, r_{n}\right)}{\log T_{f}\left(r_{1}, r_{2}, \cdots, r_{n}\right)+L\left(\frac{1}{1-r_{1}}, \frac{1}{1-r_{2}}, \cdots, \frac{1}{1-r_{n}}\right)}
$$


that is,

$$
\frac{\log T_{f \circ g}\left(r_{1}, r_{2}, \cdots, r_{n}\right)}{\log T_{f}\left(r_{1}, r_{2}, \cdots, r_{n}\right)+L\left(\frac{1}{1-r_{1}}, \frac{1}{1-r_{2}}, \cdots, \frac{1}{1-r_{n}}\right)} \geq \frac{\frac{{ }_{v_{n}} \lambda_{f \circ g}^{L^{*}}-\varepsilon}{{ }_{v_{n}} \rho_{f}^{L^{*}}+\varepsilon}}{1+\frac{L\left(\frac{1}{1-r_{1}}, \frac{1}{1-r_{2}}, \cdots, \frac{1}{1-r_{n}}\right)}{\log T_{f}\left(r_{1}, r_{2}, \cdots, r_{n}\right)}}
$$

Since $L\left(\frac{1}{1-r_{1}}, \frac{1}{1-r_{2}}, \cdots, \frac{1}{1-r_{n}}\right)=o\left\{\log T_{f}\left(r_{1}, r_{2}, \cdots, r_{n}\right)\right\}$ as $r_{1}, r_{2}, \cdots, r_{n} \rightarrow 1$, it follows from (3) that

$$
\liminf _{r_{1}, r_{2}, \cdots, r_{n} \rightarrow 1} \frac{\log T_{f \circ g}\left(r_{1}, r_{2}, \cdots, r_{n}\right)}{\log T_{f}\left(r_{1}, r_{2}, \cdots, r_{n}\right)+L\left(\frac{1}{1-r_{1}}, \frac{1}{1-r_{2}}, \cdots, \frac{1}{1-r_{n}}\right)} \geq \frac{v_{n} \lambda_{f \circ g}^{L^{*}}-\varepsilon}{v_{n} \rho_{f}^{L^{*}}+\varepsilon}
$$

As $\varepsilon(>0)$ is arbitrary, we get from above that

$$
\liminf _{r_{1}, r_{2}, \cdots, r_{n} \rightarrow 1} \frac{\log T_{f \circ g}\left(r_{1}, r_{2}, \cdots, r_{n}\right)}{\log T_{f}\left(r_{1}, r_{2}, \cdots, r_{n}\right)+L\left(\frac{1}{1-r_{1}}, \frac{1}{1-r_{2}}, \cdots, \frac{1}{1-r_{n}}\right)} \geq \frac{{ }_{v_{n}} \lambda_{f \circ g}^{L^{*}}}{v_{n} \rho_{f}^{L^{*}}}
$$

Again for a sequence of values of $\left(\frac{1}{1-r_{1}}\right),\left(\frac{1}{1-r_{2}}\right), \cdots$ and $\left(\frac{1}{1-r_{n}}\right)$ tending to infinity,

$$
\log T_{f \circ g}\left(r_{1}, r_{2}, \cdots, r_{n}\right) \leq\left({ }_{v_{n}} \lambda_{f \circ g}^{L^{*}}+\varepsilon\right) \log \left[\frac{\exp \left\{L\left(\frac{1}{1-r_{1}}, \frac{1}{1-r_{2}}, \cdots, \frac{1}{1-r_{n}}\right)\right\}}{\left(1-r_{1}\right)\left(1-r_{2}\right) \cdots\left(1-r_{n}\right)}\right]
$$

that is,

$$
\begin{aligned}
\log T_{f \circ g}\left(r_{1}, r_{2}, \cdots, r_{n}\right) & \leq\left({ }_{v_{n}} \lambda_{f \circ g}^{L^{*}}+\varepsilon\right)\left[\log \left(\frac{1}{1-r_{1}}\right)+\log \left(\frac{1}{1-r_{2}}\right), \cdots \log \left(\frac{1}{1-r_{n}}\right)\right. \\
& \left.+L\left(\frac{1}{1-r_{1}}, \frac{1}{1-r_{2}}, \cdots, \frac{1}{1-r_{n}}\right)\right]
\end{aligned}
$$

and for all sufficiently large values of $\left(\frac{1}{1-r_{1}}\right),\left(\frac{1}{1-r_{2}}\right), \cdots$ and $\left(\frac{1}{1-r_{n}}\right)$,

$$
\log T_{f}\left(r_{1}, r_{2}, \cdots, r_{n}\right) \geq\left({ }_{{ }_{n}} \lambda_{f}^{L^{*}}-\varepsilon\right) \log \left[\frac{\exp \left\{L\left(\frac{1}{1-r_{1}}, \frac{1}{1-r_{2}}, \cdots, \frac{1}{1-r_{n}}\right)\right\}}{\left(1-r_{1}\right)\left(1-r_{2}\right) \cdots\left(1-r_{n}\right)}\right]
$$

that is,

$\log T_{f}\left(r_{1}, r_{2}, \cdots, r_{n}\right) \geq\left({ }_{v_{n}} \lambda_{f}^{L^{*}}-\varepsilon\right)\left[\log \left(\frac{1}{1-r_{1}}\right)+\log \left(\frac{1}{1-r_{2}}\right), \cdots, \log \left(\frac{1}{1-r_{n}}\right)+L\left(\frac{1}{1-r_{1}}, \frac{1}{1-r_{2}}, \cdots, \frac{1}{1-r_{n}}\right)\right]$ that is,

$$
\frac{\log T_{f}\left(r_{1}, r_{2}, \cdots, r_{n}\right)}{\left({ }_{{ }_{n}} \lambda_{f}^{L^{*}}-\varepsilon\right)} \geq \log \left(\frac{1}{1-r_{1}}\right)+\log \left(\frac{1}{1-r_{2}}\right), \cdots, \log \left(\frac{1}{1-r_{n}}\right)+L\left(\frac{1}{1-r_{1}}, \frac{1}{1-r_{2}}, \cdots, \frac{1}{1-r_{n}}\right) .
$$


Combining (5) and (6), we get for a sequence of values of $\left(\frac{1}{1-r_{1}}\right),\left(\frac{1}{1-r_{2}}\right), \cdots$ and $\left(\frac{1}{1-r_{n}}\right)$ tending to infinity that

$$
\log T_{f \circ g}\left(r_{1}, r_{2}, \cdots, r_{n}\right) \leq \frac{\left({ }_{v_{n}} \lambda_{f \circ g}^{L^{*}}+\varepsilon\right)}{\left({ }_{v_{n}} \lambda_{f}^{L^{*}}-\varepsilon\right)} \log T_{f}\left(r_{1}, r_{2}, \cdots, r_{n}\right)
$$

that is,

$$
\frac{\log T_{f \circ g}\left(r_{1}, r_{2}, \cdots, r_{n}\right)}{\log T_{f}\left(r_{1}, r_{2}, \cdots, r_{n}\right)+L\left(\frac{1}{1-r_{1}}, \frac{1}{1-r_{2}}, \cdots, \frac{1}{1-r_{n}}\right)} \leq \frac{\left({ }_{{ }_{n}} \lambda_{f \circ g}^{L^{*}}+\varepsilon\right)}{\left({ }_{{ }_{n}} \lambda_{f}^{L^{*}}-\varepsilon\right)} \cdot \frac{\log T_{f}\left(r_{1}, r_{2}, \cdots, r_{n}\right)}{\log T_{f}\left(r_{1}, r_{2}, \cdots, r_{n}\right)+L\left(\frac{1}{1-r_{1}}, \frac{1}{1-r_{2}}, \cdots, \frac{1}{1-r_{n}}\right)}
$$

that is,

$$
\frac{\log T_{f \circ g}\left(r_{1}, r_{2}, \cdots, r_{n}\right)}{\log T_{f}\left(r_{1}, r_{2}, \cdots, r_{n}\right)+L\left(\frac{1}{1-r_{1}}, \frac{1}{1-r_{2}}, \cdots, \frac{1}{1-r_{n}}\right)} \leq \frac{\frac{v_{n} \lambda_{f \circ g}^{L *}+\varepsilon}{v_{n} \lambda_{f}^{L *}-\varepsilon}}{1+\frac{L\left(\frac{1}{1-r_{1}}, \frac{1}{1-r_{2}}, \cdots, \frac{1}{1-r_{n}}\right)}{\log T_{f}\left(r_{1}, r_{2}, \cdots, r_{n}\right)}}
$$

As $L\left(\frac{1}{1-r_{1}}, \frac{1}{1-r_{2}}, \cdots, \frac{1}{1-r_{n}}\right)=o\left\{\log T_{f}\left(r_{1}, r_{2}, \cdots, r_{n}\right)\right\}$ as $r_{1}, r_{2}, \cdots, r_{n} \rightarrow 1$ we get from (7) that

$$
\liminf _{r_{1}, r_{2}, \cdots, r_{n} \rightarrow 1} \frac{\log T_{f \circ g}\left(r_{1}, r_{2}, \cdots, r_{n}\right)}{\log T_{f}\left(r_{1}, r_{2}, \cdots, r_{n}\right)+L\left(\frac{1}{1-r_{1}}, \frac{1}{1-r_{2}}, \cdots, \frac{1}{1-r_{n}}\right)} \leq \frac{{ }_{v_{n}} \lambda_{f \circ g}^{L^{*}}+\varepsilon}{{ }_{v_{n}} \lambda_{f}^{L^{*}}-\varepsilon} .
$$

Since $\varepsilon(>0)$ is arbitrary, it follows from above that

$$
\liminf _{r_{1}, r_{2}, \cdots, r_{n} \rightarrow 1} \frac{\log T_{f \circ g}\left(r_{1}, r_{2}, \cdots, r_{n}\right)}{\log T_{f}\left(r_{1}, r_{2}, \cdots, r_{n}\right)+L\left(\frac{1}{1-r_{1}}, \frac{1}{1-r_{2}}, \cdots, \frac{1}{1-r_{n}}\right)} \leq \frac{{ }_{n} \lambda_{f \circ g}^{L^{*}}}{{ }_{v_{n}} \lambda_{f}^{L^{*}}} .
$$

Also for a sequence of values of $\left(\frac{1}{1-r_{1}}\right),\left(\frac{1}{1-r_{2}}\right), \cdots$ and $\left(\frac{1}{1-r_{n}}\right)$ tending to infinity,

$$
\log T_{f}\left(r_{1}, r_{2}, \cdots, r_{n}\right) \leq\left({ }_{v_{n}} \lambda_{f}^{L^{*}}+\varepsilon\right) \log \left[\frac{\exp \left\{L\left(\frac{1}{1-r_{1}}, \frac{1}{1-r_{2}}, \cdots, \frac{1}{1-r_{n}}\right)\right\}}{\left(1-r_{1}\right)\left(1-r_{2}\right) \cdots\left(1-r_{n}\right)}\right]
$$

that is,

$\log T_{f}\left(r_{1}, r_{2}, \cdots, r_{n}\right) \leq\left({ }_{v_{n}} \lambda_{f}^{L^{*}}+\varepsilon\right)\left[\log \left(\frac{1}{1-r_{1}}\right)+\log \left(\frac{1}{1-r_{2}}\right), \cdots, \log \left(\frac{1}{1-r_{n}}\right)+L\left(\frac{1}{1-r_{1}}, \frac{1}{1-r_{2}}, \cdots, \frac{1}{1-r_{n}}\right)\right]$

that is

$$
\frac{\log T_{f}\left(r_{1}, r_{2}, \cdots, r_{n}\right)}{\left(v_{n} \lambda_{f}^{L^{*}}+\varepsilon\right)} \leq \log \left(\frac{1}{1-r_{1}}\right)+\log \left(\frac{1}{1-r_{2}}\right), \cdots \log ,\left(\frac{1}{1-r_{n}}\right)+L\left(\frac{1}{1-r_{1}}, \frac{1}{1-r_{2}}, \cdots, \frac{1}{1-r_{n}}\right) .
$$

Now from (1) and (9), we obtain for a sequence of values of $\left(\frac{1}{1-r_{1}}\right),\left(\frac{1}{1-r_{2}}\right), \cdots$ and $\left(\frac{1}{1-r_{n}}\right)$ tending to infinity that

$$
\log T_{f \circ g}\left(r_{1}, r_{2}, \cdots, r_{n}\right) \geq \frac{\left({ }_{v_{n}} \lambda_{f \circ g}^{L^{*}}-\varepsilon\right)}{\left({ }_{v_{n}} \lambda_{f}^{L^{*}}+\varepsilon\right)} \log T_{f}\left(r_{1}, r_{2}, \cdots, r_{n}\right)
$$


that is,

$$
\frac{\log T_{f \circ g}\left(r_{1}, r_{2}, \cdots, r_{n}\right)}{\log T_{f}\left(r_{1}, r_{2}, \cdots, r_{n}\right)+L\left(\frac{1}{1-r_{1}}, \frac{1}{1-r_{2}}, \cdots, \frac{1}{1-r_{n}}\right)} \geq \frac{\left(v_{n} \lambda_{f \circ g}^{L^{*}}-\varepsilon\right)}{\left({ }_{v_{n}} \lambda_{f}^{L^{*}}+\varepsilon\right)} \cdot \frac{\log T_{f}\left(r_{1}, r_{2}, \cdots, r_{n}\right)}{\log T_{f}\left(r_{1}, r_{2}, \cdots, r_{n}\right)+L\left(\frac{1}{1-r_{1}}, \frac{1}{1-r_{2}}, \cdots, \frac{1}{1-r_{n}}\right)}
$$

that is,

$$
\frac{\log T_{f \circ g}\left(r_{1}, r_{2}, \cdots, r_{n}\right)}{\log T_{f}\left(r_{1}, r_{2}, \cdots, r_{n}\right)+L\left(\frac{1}{1-r_{1}}, \frac{1}{1-r_{2}}, \cdots, \frac{1}{1-r_{n}}\right)} \geq \frac{\frac{v_{n} \lambda_{f \circ g}^{L^{*}}-\varepsilon}{{ }_{v_{n}} \lambda_{f}^{L^{*}}+\varepsilon}}{1+\frac{L\left(\frac{1}{1-r_{1}}, \frac{1}{1-r_{2}}, \cdots, \frac{1}{1-r_{n}}\right)}{\log T_{f}\left(r_{1}, r_{2}, \cdots, r_{n}\right)}}
$$

In view of the condition $L\left(\frac{1}{1-r_{1}}, \frac{1}{1-r_{2}}, \cdots, \frac{1}{1-r_{n}}\right)=o\left\{\log T_{f}\left(r_{1}, r_{2}, \cdots, r_{n}\right)\right\}$ as $r_{1}, r_{2}, \cdots, r_{n} \rightarrow 1$, we obtain from (10) that

$$
\limsup _{r_{1}, r_{2}, \cdots, r_{n} \rightarrow 1} \frac{\log T_{f \circ g}\left(r_{1}, r_{2}, \cdots, r_{n}\right)}{\log T_{f}\left(r_{1}, r_{2}, \cdots, r_{n}\right)+L\left(\frac{1}{1-r_{1}}, \frac{1}{1-r_{2}}, \cdots, \frac{1}{1-r_{n}}\right)} \geq \frac{{ }_{v_{n}} \lambda_{f \circ g}^{L^{*}}-\varepsilon}{{ }_{v_{n}} \lambda_{f}^{L^{*}}+\varepsilon}
$$

Since $\varepsilon(>0)$ is arbitrary, it follows from above that

$$
\limsup _{r_{1}, r_{2}, \cdots, r_{n} \rightarrow 1} \frac{\log T_{f \circ g}\left(r_{1}, r_{2}, \cdots, r_{n}\right)}{\log T_{f}\left(r_{1}, r_{2}, \cdots, r_{n}\right)+L\left(\frac{1}{1-r_{1}}, \frac{1}{1-r_{2}}, \cdots, \frac{1}{1-r_{n}}\right)} \geq \frac{v_{n} \lambda_{f \circ g}^{L^{*}}}{{ }_{v_{n}} \lambda_{f}^{L^{*}}} .
$$

Also for all sufficiently large values of $\left(\frac{1}{1-r_{1}}\right),\left(\frac{1}{1-r_{2}}\right), \cdots$ and $\left(\frac{1}{1-r_{n}}\right)$,

$$
\log T_{f \circ g}\left(r_{1}, r_{2}, \cdots, r_{n}\right) \leq\left({ }_{v_{n}} \rho_{f \circ g}^{L^{*}}+\varepsilon\right) \log \left[\frac{\exp \left\{L\left(\frac{1}{1-r_{1}}, \frac{1}{1-r_{2}}, \cdots, \frac{1}{1-r_{n}}\right)\right\}}{\left(1-r_{1}\right)\left(1-r_{2}\right) \cdots\left(1-r_{n}\right)}\right]
$$

that is,

$$
\begin{aligned}
\log T_{f \circ g}\left(r_{1}, r_{2}, \cdots, r_{n}\right) & \leq\left({ }_{v_{n}} \rho_{f \circ g}^{L^{*}}+\varepsilon\right)\left[\log \left(\frac{1}{1-r_{1}}\right)+\log \left(\frac{1}{1-r_{2}}\right), \cdots, \log \left(\frac{1}{1-r_{n}}\right)\right. \\
& \left.+L\left(\frac{1}{1-r_{1}}, \frac{1}{1-r_{2}}, \cdots, \frac{1}{1-r_{n}}\right)\right]
\end{aligned}
$$

So from (6) and (12), it follows for all sufficiently large values of $\left(\frac{1}{1-r_{1}}\right),\left(\frac{1}{1-r_{2}}\right), \cdots$ and $\left(\frac{1}{1-r_{n}}\right)$ that

$$
\log T_{f \circ g}\left(r_{1}, r_{2}, \cdots, r_{n}\right) \leq \frac{\left({ }_{v_{n}} \rho_{f \circ g}^{L^{*}}+\varepsilon\right)}{\left({ }_{v_{n}} \lambda_{f}^{L^{*}}-\varepsilon\right)} \log T_{f}\left(r_{1}, r_{2}, \cdots, r_{n}\right)
$$

that is,

$$
\frac{\log T_{f \circ g}\left(r_{1}, r_{2}, \cdots, r_{n}\right)}{\log T_{f}\left(r_{1}, r_{2}, \cdots, r_{n}\right)+L\left(\frac{1}{1-r_{1}}, \frac{1}{1-r_{2}}, \cdots, \frac{1}{1-r_{n}}\right)} \leq \frac{\left(v_{n} \rho_{f \circ g}^{L^{*}}+\varepsilon\right)}{\left({ }_{v_{n}} \lambda_{f}^{L^{*}}-\varepsilon\right)} \cdot \frac{\log T_{f}\left(r_{1}, r_{2}, \cdots, r_{n}\right)}{\log T_{f}\left(r_{1}, r_{2}, \cdots, r_{n}\right)+L\left(\frac{1}{1-r_{1}}, \frac{1}{1-r_{2}}, \cdots, \frac{1}{1-r_{n}}\right)}
$$


that is,

$$
\frac{\log T_{f \circ g}\left(r_{1}, r_{2}, \cdots, r_{n}\right)}{\log T_{f}\left(r_{1}, r_{2}, \cdots, r_{n}\right)+L\left(\frac{1}{1-r_{1}}, \frac{1}{1-r_{2}}, \cdots, \frac{1}{1-r_{n}}\right)} \leq \frac{\frac{v_{n} \rho_{f \circ g}^{L^{*}}+\varepsilon}{v_{n} \lambda_{f}^{L^{*}}-\varepsilon}}{1+\frac{L\left(\frac{1}{1-r_{1}}, \frac{1}{1-r_{2}}, \cdots, \frac{1}{1-r_{n}}\right)}{\log T_{f}\left(r_{1}, r_{2}, \cdots, r_{n}\right)}}
$$

Using $L\left(\frac{1}{1-r_{1}}, \frac{1}{1-r_{2}}, \cdots, \frac{1}{1-r_{n}}\right)=o\left\{\log T_{f}\left(r_{1}, r_{2}, \cdots, r_{n}\right)\right\}$ as $r_{1}, r_{2}, \cdots, r_{n} \rightarrow 1$, we obtain from (13) that

$$
\limsup _{r_{1}, r_{2}, \cdots, r_{n} \rightarrow 1} \frac{\log T_{f \circ g}\left(r_{1}, r_{2}, \cdots, r_{n}\right)}{\log T_{f}\left(r_{1}, r_{2}, \cdots, r_{n}\right)+L\left(\frac{1}{1-r_{1}}, \frac{1}{1-r_{2}}, \cdots, \frac{1}{1-r_{n}}\right)} \leq \frac{v_{n} \rho_{f \circ g}^{L^{*}}+\varepsilon}{v_{n} \lambda_{f}^{L^{*}}-\varepsilon} .
$$

As $\varepsilon(>0)$ is arbitrary, it follows from above that

$$
\limsup _{r_{1}, r_{2}, \cdots, r_{n} \rightarrow 1} \frac{\log T_{f \circ g}\left(r_{1}, r_{2}, \cdots, r_{n}\right)}{\log T_{f}\left(r_{1}, r_{2}, \cdots, r_{n}\right)+L\left(\frac{1}{1-r_{1}}, \frac{1}{1-r_{2}}, \cdots, \frac{1}{1-r_{n}}\right)} \leq \frac{v_{n} \rho_{f \circ g}^{L^{*}}}{{ }_{v_{n}} \lambda_{f}^{L^{*}}}
$$

Thus the theorem follows from $(4),(8),(11)$ and (14).

Similarly in view of Theorem 1 , we may state the following theorem without its proof for the right factor $g$ of the composite function $f \circ g$ :

Theorem 2. Let $f$ and $g$ be any two non-constant analytic functions of $n$ complex variables in the unit polydisc $U$ such that $0<{ }_{v_{n}} \lambda_{f \circ g}^{L^{*}} \leq{ }_{v_{n}} \rho_{f \circ g}^{L^{*}}<\infty$ and $0<{ }_{v_{n}} \lambda_{g}^{L^{*}} \leq{ }_{v_{n}} \rho_{g}^{L^{*}}<\infty$. If $L\left(\frac{1}{1-r_{1}}, \frac{1}{1-r_{2}}, \cdots, \frac{1}{1-r_{n}}\right)=o\left\{\log T_{g}\left(r_{1}, r_{2}, \cdots, r_{n}\right)\right\}$ as $r_{1}, r_{2}, \cdots, r_{n} \rightarrow 1$ then

$$
\begin{aligned}
\frac{v_{n}}{v_{f} \rho_{g}^{L^{*}}} & \leq \liminf _{v_{1}, r_{2}, \cdots, r_{n} \rightarrow 1} \frac{\log T_{f \circ g}\left(r_{1}, r_{2}, \cdots, r_{n}\right)}{\log T_{g}\left(r_{1}, r_{2}, \cdots, r_{n}\right)+L\left(\frac{1}{1-r_{1}}, \frac{1}{1-r_{2}}, \cdots, \frac{1}{1-r_{n}}\right)} \leq \frac{{ }_{v_{n}} \lambda_{f \circ g}^{L^{*}}}{{ }_{v_{n}} \lambda_{g}^{L^{*}}} \\
& \leq \limsup _{r_{1}, r_{2}, \cdots, r_{n} \rightarrow 1} \frac{\log T_{f \circ g}\left(r_{1}, r_{2}, \cdots, r_{n}\right)}{\log T_{g}\left(r_{1}, r_{2}, \cdots, r_{n}\right)+L\left(\frac{1}{1-r_{1}}, \frac{1}{1-r_{2}}, \cdots, \frac{1}{1-r_{n}}\right)} \leq \frac{{ }_{v_{n}} \rho_{f \circ g}^{L^{*}}}{{ }_{v_{n}} \lambda_{g}^{L^{*}}}
\end{aligned}
$$

Theorem 3. Let $f$ and $g$ be any two non-constant analytic functions of $n$ complex variables in the unit polydisc $U$ such that $0<{ }_{v_{n}} \rho_{f \circ g}^{L^{*}}<\infty$ and $0<{ }_{v_{n}} \rho_{f}^{L^{*}}<\infty$. If $L\left(\frac{1}{1-r_{1}}, \frac{1}{1-r_{2}}, \cdots, \frac{1}{1-r_{n}}\right)=o\left\{\log T_{f}\left(r_{1}, r_{2}, \cdots, r_{n}\right)\right\}$ as $r_{1}, r_{2}, \cdots, r_{n} \rightarrow 1$ then

$$
\begin{aligned}
\liminf _{r_{1}, r_{2}, \cdots, r_{n} \rightarrow 1} \frac{\log T_{f \circ g}\left(r_{1}, r_{2}, \cdots, r_{n}\right)}{\log T_{f}\left(r_{1}, r_{2}, \cdots, r_{n}\right)+L\left(\frac{1}{1-r_{1}}, \frac{1}{1-r_{2}}, \cdots, \frac{1}{1-r_{n}}\right)} & \leq \frac{v_{n} \rho_{f \circ g}^{L^{*}}}{{ }_{v_{n}} \rho_{f}^{L^{*}}} \\
& \leq \limsup _{r_{1}, r_{2}, \cdots, r_{n} \rightarrow 1} \frac{\log T_{f \circ g}\left(r_{1}, r_{2}, \cdots, r_{n}\right)}{\log T_{f}\left(r_{1}, r_{2}, \cdots, r_{n}\right)+L\left(\frac{1}{1-r_{1}}, \frac{1}{1-r_{2}}, \cdots, \frac{1}{1-r_{n}}\right)}
\end{aligned}
$$

Proof. From the definition of ${ }_{v_{n}} \rho_{f}^{L^{*}}$, the Nevanlinna $n$ variables based $L^{*}$-order of analytic function $f$ in the unit disc $U$, we get for a sequence of values of $\left(\frac{1}{1-r_{1}}\right),\left(\frac{1}{1-r_{2}}\right), \cdots$, and $\left(\frac{1}{1-r_{n}}\right)$ tending to infinity that

$$
\log T_{f}\left(r_{1}, r_{2}, \cdots, r_{n}\right) \geq\left({ }_{v} \rho_{f}^{L^{*}}-\varepsilon\right) \log \left[\frac{\exp \left\{L\left(\frac{1}{1-r_{1}}, \frac{1}{1-r_{2}}, \cdots, \frac{1}{1-r_{n}}\right)\right\}}{\left(1-r_{1}\right)\left(1-r_{2}\right) \cdots\left(1-r_{n}\right)}\right]
$$


that is,

$$
\log T_{f}\left(r_{1}, r_{2}, \cdots, r_{n}\right) \geq\left({ }_{v_{n}} \rho_{f}^{L^{*}}-\varepsilon\right)\left[\log \left(\frac{1}{1-r_{1}}\right)+\log \left(\frac{1}{1-r_{2}}\right), \cdots, \log \left(\frac{1}{1-r_{n}}\right)+L\left(\frac{1}{1-r_{1}}, \frac{1}{1-r_{2}}, \cdots, \frac{1}{1-r_{n}}\right)\right]
$$

that is,

$$
\frac{\log T_{f}\left(r_{1}, r_{2}, \cdots, r_{n}\right)}{\left(v_{n} \rho_{f}^{L^{*}}-\varepsilon\right)} \geq \log \left(\frac{1}{1-r_{1}}\right)+\log \left(\frac{1}{1-r_{2}}\right), \cdots \log \left(\frac{1}{1-r_{n}}\right)+L\left(\frac{1}{1-r_{1}}, \frac{1}{1-r_{2}}, \cdots, \frac{1}{1-r_{n}}\right)
$$

Now from (12) and (15), it follows for a sequence of values of $\left(\frac{1}{1-r_{1}}\right),\left(\frac{1}{1-r_{2}}\right), \cdots$ and $\left(\frac{1}{1-r_{n}}\right)$ tending to infinity that

$$
\log T_{f \circ g}\left(r_{1}, r_{2}, \cdots, r_{n}\right) \leq \frac{\left(v_{n} \rho_{f \circ g}^{L^{*}}+\varepsilon\right)}{\left({ }_{v_{n}} \rho_{f}^{L^{*}}-\varepsilon\right)} \log T_{f}\left(r_{1}, r_{2}, \cdots, r_{n}\right)
$$

that is,

$$
\frac{\log T_{f \circ g}\left(r_{1}, r_{2}, \cdots, r_{n}\right)}{\log T_{f}\left(r_{1}, r_{2}, \cdots, r_{n}\right)+L\left(\frac{1}{1-r_{1}}, \frac{1}{1-r_{2}}, \cdots, \frac{1}{1-r_{n}}\right)} \leq \frac{\left({ }_{n} \rho_{f \circ g}^{L^{*}}+\varepsilon\right)}{\left(v_{n} \rho_{f}^{L^{*}}-\varepsilon\right)} \cdot \frac{\log T_{f}\left(r_{1}, r_{2}, \cdots, r_{n}\right)}{\log T_{f}\left(r_{1}, r_{2}, \cdots, r_{n}\right)+L\left(\frac{1}{1-r_{1}}, \frac{1}{1-r_{2}}, \cdots, \frac{1}{1-r_{n}}\right)}
$$

Thus,

$$
\frac{\log T_{f \circ g}\left(r_{1}, r_{2}, \cdots, r_{n}\right)}{\log T_{f}\left(r_{1}, r_{2}, \cdots, r_{n}\right)+L\left(\frac{1}{1-r_{1}}, \frac{1}{1-r_{2}}, \cdots, \frac{1}{1-r_{n}}\right)} \leq \frac{\frac{v_{n} \rho_{f \circ g}^{L^{*}}+\varepsilon}{v_{n} \rho_{f}^{L^{*}}-\varepsilon}}{1+\frac{L\left(\frac{1}{1-r_{1}}, \frac{1}{1-r_{2}}, \cdots, \frac{1}{1-r_{n}}\right)}{\log T_{f}\left(r_{1}, r_{2}, \cdots, r_{n}\right)}}
$$

Using $L\left(\frac{1}{1-r_{1}}, \frac{1}{1-r_{2}}, \cdots, \frac{1}{1-r_{n}}\right)=o\left\{\log T_{f}\left(r_{1}, r_{2}, \cdots, r_{n}\right)\right\}$ as $r_{1}, r_{2}, \cdots, r_{n} \rightarrow 1$, we obtain from (16) that

$$
\liminf _{r_{1}, r_{2}, \cdots, r_{n} \rightarrow 1} \frac{\log T_{f \circ g}\left(r_{1}, r_{2}, \cdots, r_{n}\right)}{\log T_{f}\left(r_{1}, r_{2}, \cdots, r_{n}\right)+L\left(\frac{1}{1-r_{1}}, \frac{1}{1-r_{2}}, \cdots, \frac{1}{1-r_{n}}\right)} \leq \frac{v_{n} \rho_{f \circ g}^{L^{*}}+\varepsilon}{v_{n} \rho_{f}^{L^{*}}-\varepsilon}
$$

As $\varepsilon(>0)$ is arbitrary, it follows from above that

$$
\liminf _{r_{1}, r_{2}, \cdots, r_{n} \rightarrow 1} \frac{\log T_{f \circ g}\left(r_{1}, r_{2}, \cdots, r_{n}\right)}{\log T_{f}\left(r_{1}, r_{2}, \cdots, r_{n}\right)+L\left(\frac{1}{1-r_{1}}, \frac{1}{1-r_{2}}, \cdots, \frac{1}{1-r_{n}}\right)} \leq \frac{v_{n} \rho_{f \circ g}^{L^{*}}}{v_{n} \rho_{f}^{L^{*}}}
$$

Again for a sequence of values of $\left(\frac{1}{1-r_{1}}\right),\left(\frac{1}{1-r_{2}}\right), \cdots$ and $\left(\frac{1}{1-r_{n}}\right)$ tending to infinity,

$$
\log T_{f \circ g}\left(r_{1}, r_{2}, \cdots, r_{n}\right) \geq\left({ }_{v} \rho_{f \circ g}^{L^{*}}-\varepsilon\right) \log \left[\frac{\exp \left\{L\left(\frac{1}{1-r_{1}}, \frac{1}{1-r_{2}}, \cdots, \frac{1}{1-r_{n}}\right)\right\}}{\left(1-r_{1}\right)\left(1-r_{2}\right) \cdots\left(1-r_{n}\right)}\right]
$$


that is,

$$
\begin{aligned}
\log T_{f \circ g}\left(r_{1}, r_{2}, \cdots, r_{n}\right) & \geq\left({ }_{v_{n}} \rho_{f \circ g}^{[m] L^{*}}-\varepsilon\right)\left[\log \left(\frac{1}{1-r_{1}}\right)+\log \left(\frac{1}{1-r_{2}}\right), \cdots, \log \left(\frac{1}{1-r_{n}}\right)\right. \\
& \left.+L\left(\frac{1}{1-r_{1}}, \frac{1}{1-r_{2}}, \cdots, \frac{1}{1-r_{n}}\right)\right]
\end{aligned}
$$

So combining (2) and (18), we get for a sequence of values of $\left(\frac{1}{1-r_{1}}\right),\left(\frac{1}{1-r_{2}}\right), \cdots$ and $\left(\frac{1}{1-r_{n}}\right)$ tending to infinity that

$$
\log T_{f \circ g}\left(r_{1}, r_{2}, \cdots, r_{n}\right) \geq \frac{\left({ }_{v_{n}} \rho_{f \circ g}^{L^{*}}-\varepsilon\right)}{\left({ }_{v_{n}} \rho_{f}^{L^{*}}+\varepsilon\right)} \log T_{f}\left(r_{1}, r_{2}, \cdots, r_{n}\right)
$$

that is,

$$
\frac{\log T_{f \circ g}\left(r_{1}, r_{2}, \cdots, r_{n}\right)}{\log T_{f}\left(r_{1}, r_{2}, \cdots, r_{n}\right)+L\left(\frac{1}{1-r_{1}}, \frac{1}{1-r_{2}}, \cdots, \frac{1}{1-r_{n}}\right)} \geq \frac{\left(v_{n} \rho_{f \circ g}^{L^{*}}-\varepsilon\right)}{\left({ }_{n} \rho_{f}^{L^{*}}+\varepsilon\right)} \cdot \frac{\log T_{f}\left(r_{1}, r_{2}, \cdots, r_{n}\right)}{\log T_{f}\left(r_{1}, r_{2}, \cdots, r_{n}\right)+L\left(\frac{1}{1-r_{1}}, \frac{1}{1-r_{2}}, \cdots, \frac{1}{1-r_{n}}\right)} .
$$

Thus,

$$
\frac{\log T_{f \circ g}\left(r_{1}, r_{2}, \cdots, r_{n}\right)}{\log T_{f}\left(r_{1}, r_{2}, \cdots, r_{n}\right)+L\left(\frac{1}{1-r_{1}}, \frac{1}{1-r_{2}}, \cdots, \frac{1}{1-r_{n}}\right)} \geq \frac{\frac{v_{n} \rho_{f \circ g}^{L^{*}}-\varepsilon}{v_{n} \rho_{f}^{L^{*}+\varepsilon}}}{1+\frac{L\left(\frac{1}{1-r_{1},}, \frac{1}{\left.1-r_{2}, \cdots, \frac{1}{1-r_{n}}\right)}\right.}{\log T_{f}\left(r_{1}, r_{2}, \cdots, r_{n}\right)}}
$$

Since $L\left(\frac{1}{1-r_{1}}, \frac{1}{1-r_{2}}, \cdots, \frac{1}{1-r_{n}}\right)=o\left\{\log T_{f}\left(r_{1}, r_{2}, \cdots, r_{n}\right)\right\}$ as $r_{1}, r_{2}, \cdots, r_{n} \rightarrow 1$, it follows from (19) that

$$
\limsup _{r_{1}, r_{2}, \cdots, r_{n} \rightarrow 1} \frac{\log T_{f \circ g}\left(r_{1}, r_{2}, \cdots, r_{n}\right)}{\log T_{f}\left(r_{1}, r_{2}, \cdots, r_{n}\right)+L\left(\frac{1}{1-r_{1}}, \frac{1}{1-r_{2}}, \cdots, \frac{1}{1-r_{n}}\right)} \geq \frac{v_{n} \rho_{f \circ g}^{L^{*}}-\varepsilon}{v_{n} \rho_{f}^{L^{*}}+\varepsilon}
$$

As $\varepsilon(>0)$ is arbitrary, we get from above that

$$
\limsup _{r_{1}, r_{2}, \cdots, r_{n} \rightarrow 1} \frac{\log T_{f \circ g}\left(r_{1}, r_{2}, \cdots, r_{n}\right)}{\log T_{f}\left(r_{1}, r_{2}, \cdots, r_{n}\right)+L\left(\frac{1}{1-r_{1}}, \frac{1}{1-r_{2}}, \cdots, \frac{1}{1-r_{n}}\right)} \geq \frac{{ }_{n} \rho_{f \circ g}^{L^{*}}}{{ }_{v_{n}} \rho_{f}^{L^{*}}} .
$$

Thus the theorem follows from (17) and (20).

Theorem 4. Let $f$ and $g$ be any two non-constant analytic functions of $n$ complex variables in the unit polydisc $U$ such that $0<{ }_{v_{n}} \rho_{\text {fog }}^{L^{*}}<\infty$ and $0<{ }_{v_{n}} \rho_{g}^{L^{*}}<\infty$. If $L\left(\frac{1}{1-r_{1}}, \frac{1}{1-r_{2}}, \cdots, \frac{1}{1-r_{n}}\right)=o\left\{\log T_{g}\left(r_{1}, r_{2}, \cdots, r_{n}\right)\right\}$ as $r_{1}, r_{2}, \cdots, r_{n} \rightarrow 1$ then

$$
\begin{aligned}
\liminf _{r_{1}, r_{2}, \cdots, r_{n} \rightarrow 1} \frac{\log T_{f \circ g}\left(r_{1}, r_{2}, \cdots, r_{n}\right)}{\log T_{g}\left(r_{1}, r_{2}, \cdots, r_{n}\right)+L\left(\frac{1}{1-r_{1}}, \frac{1}{1-r_{2}}, \cdots, \frac{1}{1-r_{n}}\right)} & \leq \frac{v_{n} \rho_{f \circ g}^{L^{*}}}{v_{n} \rho_{g}^{L^{*}}} \\
& \leq \limsup _{r_{1}, r_{2}, \cdots, r_{n} \rightarrow 1} \frac{\log T_{f \circ g}\left(r_{1}, r_{2}, \cdots, r_{n}\right)}{\log T_{g}\left(r_{1}, r_{2}, \cdots, r_{n}\right)+L\left(\frac{1}{1-r_{1}}, \frac{1}{1-r_{2}}, \cdots, \frac{1}{1-r_{n}}\right)} .
\end{aligned}
$$

The proof is omitted. The following theorem is a natural consequence of Theorem 1 and Theorem 3. 
Theorem 5. Let $f$ and $g$ be any two non-constant analytic functions of $n$ complex variables in the unit polydisc $U$ such that $0<{ }_{v_{n}} \lambda_{f \circ g}^{L^{*}} \leq v_{n} \rho_{f \circ g}^{L^{*}}<\infty$ and $0<{ }_{v_{n}} \lambda_{f}^{L^{*}} \leq{ }_{v_{n}} \rho_{f}^{L^{*}}<\infty$. If $L\left(\frac{1}{1-r_{1}}, \frac{1}{1-r_{2}}, \cdots, \frac{1}{1-r_{n}}\right)=o\left\{\log T_{f}\left(r_{1}, r_{2}, \cdots, r_{n}\right)\right\}$ as $r_{1}, r_{2}, \cdots, r_{n} \rightarrow 1$ then

$$
\begin{aligned}
& \liminf _{r_{1}, r_{2}, \cdots, r_{n} \rightarrow 1} \frac{\log T_{f \circ g}\left(r_{1}, r_{2}, \cdots, r_{n}\right)}{\log T_{f}\left(r_{1}, r_{2}, \cdots, r_{n}\right)+L\left(\frac{1}{1-r_{1}}, \frac{1}{1-r_{2}}, \cdots, \frac{1}{1-r_{n}}\right)} \leq \min \left\{\frac{{ }_{v_{n}} \lambda_{f \circ g}^{L^{*}}}{v_{n} \lambda_{f}^{L^{*}}}, \frac{v_{n} \rho_{f \circ g}^{L^{*}}}{{ }_{v_{n}} \rho_{f}^{L^{*}}}\right\} \\
& \leq \max \left\{\frac{v_{n} \lambda_{f \circ g}^{L^{*}}}{{ }_{v_{n}} \lambda_{f}^{L^{*}}}, \frac{v_{n} \rho_{f \circ g}^{L^{*}}}{v_{n} \rho_{f}^{L^{*}}}\right\} \leq \limsup _{r_{1}, r_{2}, \cdots, r_{n} \rightarrow 1} \frac{\log T_{f \circ g}\left(r_{1}, r_{2}, \cdots, r_{n}\right)}{\log T_{f}\left(r_{1}, r_{2}, \cdots, r_{n}\right)+L\left(\frac{1}{1-r_{1}}, \frac{1}{1-r_{2}}, \cdots, \frac{1}{1-r_{n}}\right)}
\end{aligned}
$$

The proof is omitted. Combining Theorem 2 and Theorem 4, we may state the following theorem:

Theorem 6. Let $f$ and $g$ be any two non-constant analytic functions of $n$ complex variables in the unit polydisc $U$ such that $0<{ }_{v_{n}} \lambda_{f \circ g}^{L^{*}} \leq{ }_{v_{n}} \rho_{f \circ g}^{L^{*}}<\infty$ and $0<{ }_{v_{n}} \lambda_{g}^{L^{*}} \leq{ }_{v_{n}} \rho_{g}^{L^{*}}<\infty$. If $L\left(\frac{1}{1-r_{1}}, \frac{1}{1-r_{2}}, \cdots, \frac{1}{1-r_{n}}\right)=o\left\{\log T_{g}\left(r_{1}, r_{2}, \cdots, r_{n}\right)\right\}$ as $r_{1}, r_{2}, \cdots, r_{n} \rightarrow 1$ then

$$
\begin{aligned}
& \liminf _{r_{1}, r_{2}, \cdots, r_{n} \rightarrow 1} \frac{\log T_{f \circ g}\left(r_{1}, r_{2}, \cdots, r_{n}\right)}{\log T_{g}\left(r_{1}, r_{2}, \cdots, r_{n}\right)+L\left(\frac{1}{1-r_{1}}, \frac{1}{1-r_{2}}, \cdots, \frac{1}{1-r_{n}}\right)} \leq \min \left\{\frac{{ }_{v_{n}} \lambda_{f \circ g}^{L^{*}}}{v_{n} \lambda_{g}^{L^{*}}}, \frac{v_{n} \rho_{f \circ g}^{L^{*}}}{v_{n} \rho_{g}^{L^{*}}}\right\} \\
& \leq \max \left\{\frac{v_{n} \lambda \lambda_{f \circ g}^{L^{*}}}{v_{n} \lambda_{g}^{L^{*}}}, \frac{v_{n} \rho_{f \circ g}^{L^{*}}}{v_{n} \rho_{g}^{L^{*}}}\right\} \leq \limsup _{r_{1}, r_{2}, \cdots, r_{n} \rightarrow 1} \frac{\log T_{f \circ g}\left(r_{1}, r_{2}, \cdots, r_{n}\right)}{\log T_{g}\left(r_{1}, r_{2}, \cdots, r_{n}\right)+L\left(\frac{1}{1-r_{1}}, \frac{1}{1-r_{2}}, \cdots, \frac{1}{1-r_{n}}\right)}
\end{aligned}
$$

Theorem 7. Let $f$ be a non-constant analytic function of $n$ complex variables in the unit polydisc $U$ with $v_{n} \rho_{f}^{L^{*}}<\infty$. Also let $g$ be a non-constant analytic function of $n$ complex variables in the unit polydisc $U . I f_{v_{n}} \lambda_{f \circ g}^{L^{*}}=\infty$ then

$$
\lim _{r_{1}, r_{2}, \cdots, r_{n} \rightarrow 1} \frac{\log T_{f \circ g}\left(r_{1}, r_{2}, \cdots, r_{n}\right)}{\log T_{f}\left(r_{1}, r_{2}, \cdots, r_{n}\right)}=\infty
$$

Proof. Let us suppose that the conclusion of the theorem do not hold.Then we can find a constant $\beta>0$ such that for a sequence of values of $\left(\frac{1}{1-r_{1}}\right),\left(\frac{1}{1-r_{2}}\right), \cdots$ and $\left(\frac{1}{1-r_{n}}\right)$ tending to infinity that

$$
\log T_{f \circ g}\left(r_{1}, r_{2}, \cdots, r_{n}\right) \leq \beta \log T_{f}\left(r_{1}, r_{2}, \cdots, r_{n}\right) .
$$

Again from the definition of ${ }_{v_{n}} \rho_{f}^{L^{*}}$, it follows that for all sufficiently large values of $\left(\frac{1}{1-r_{1}}\right),\left(\frac{1}{1-r_{2}}\right), \cdots$ and $\left(\frac{1}{1-r_{n}}\right)$ that

$$
\log T_{f}\left(r_{1}, r_{2}, \cdots, r_{n}\right) \leq\left({ }_{n} \rho_{f}^{L^{*}}+\varepsilon\right) \log \left[\frac{\exp \left\{L\left(\frac{1}{1-r_{1}}, \frac{1}{1-r_{2}}, \cdots, \frac{1}{1-r_{n}}\right)\right\}}{\left(1-r_{1}\right)\left(1-r_{2}\right) \cdots\left(1-r_{n}\right)}\right]
$$

Thus from (21) and above, we have for a sequence of values of $\left(\frac{1}{1-r_{1}}\right),\left(\frac{1}{1-r_{2}}\right), \cdots$ and $\left(\frac{1}{1-r_{n}}\right)$ tending to infinity that

$$
\log T_{f \circ g}\left(r_{1}, r_{2}, \cdots, r_{n}\right) \leq \beta\left({ }_{v_{n}} \rho_{f}^{L^{*}}+\varepsilon\right) \log \left[\frac{\exp \left\{L\left(\frac{1}{1-r_{1}}, \frac{1}{1-r_{2}}, \cdots, \frac{1}{1-r_{n}}\right)\right\}}{\left(1-r_{1}\right)\left(1-r_{2}\right) \cdots\left(1-r_{n}\right)}\right]
$$


that is,

$$
\frac{\log T_{f \circ g}\left(r_{1}, r_{2}, \cdots, r_{n}\right)}{\log \left[\frac{\exp \left\{L\left(\frac{1}{1-r_{1}}, \frac{1}{1-r_{2}}, \cdots, \frac{1}{1-r_{n}}\right)\right\}}{\left(1-r_{1}\right)\left(1-r_{2}\right) \cdots\left(1-r_{n}\right)}\right]} \leq \frac{\beta\left({ }_{n} \rho_{f}^{L^{*}}+\varepsilon\right) \log \left[\frac{\exp \left\{L\left(\frac{1}{1-r_{1}}, \frac{1}{1-r_{2}}, \cdots, \frac{1}{1-r_{n}}\right)\right\}}{\left(1-r_{1}\right)\left(1-r_{2}\right) \cdots\left(1-r_{n}\right)}\right]}{\log \left[\frac{\exp \left\{L\left(\frac{1}{1-r_{1}}, \frac{1}{1-r_{2}}, \cdots, \frac{1}{1-r_{n}}\right)\right\}}{\left(1-r_{1}\right)\left(1-r_{2}\right) \cdots\left(1-r_{n}\right)}\right]}
$$

Therefore,

$$
\liminf _{r_{1}, r_{2}, \cdots, r_{n} \rightarrow 1} \frac{\log T_{f \circ g}\left(r_{1}, r_{2}, \cdots, r_{n}\right)}{\log \left[\frac{\exp \left\{L\left(\frac{1}{1-r_{1}}, \frac{1}{1-r_{2}}, \cdots, \frac{1}{1-r_{n}}\right)\right\}}{\left(1-r_{1}\right)\left(1-r_{2}\right) \cdots\left(1-r_{n}\right)}\right]}=v_{n} \lambda_{f \circ g}^{L^{*}}<\infty .
$$

This is a contradiction. This proves the theorem.

Remark. Theorem 7 is also valid with "limit superior" instead of "limit" if ${ }_{v_{n}} \lambda_{f \circ g}^{L^{*}}=\infty$ is replaced by $v_{n} \rho_{f \circ g}^{L^{*}}=\infty$ and the other conditions remaining the same.

Corollary 1. Under the assumptions of Theorem 7 or Remark 2,

$$
\lim _{r_{1}, r_{2}, \cdots, r_{n} \rightarrow 1} \frac{T_{f \circ g}\left(r_{1}, r_{2}, \cdots, r_{n}\right)}{T_{f}\left(r_{1}, r_{2}, \cdots, r_{n}\right)}=\infty .
$$

Proof. From Theorem 7 or Remark 2, we obtain for all sufficiently large values of $\left(\frac{1}{1-r}\right)$ and for $K>1$ that

$$
\begin{aligned}
\log T_{f \circ g}\left(r_{1}, r_{2}, \cdots, r_{n}\right) & >K T_{f}\left(r_{1}, r_{2}, \cdots, r_{n}\right), \\
T_{f \circ g}\left(r_{1}, r_{2}, \cdots, r_{n}\right) & >\left\{T_{f}\left(r_{1}, r_{2}, \cdots, r_{n}\right)\right\}^{K},
\end{aligned}
$$

from which the corollary follows.

Theorem 8. Let $f$ and $g$ be any two non-constant analytic functions of $n$ complex variables in the unit polydisc $U$ such that $_{v_{n}} \rho_{g}^{L^{*}}<\infty$ and $_{v_{n}} \lambda_{f \circ g}^{L^{*}}=\infty$. Then

$$
\lim _{r_{1}, r_{2}, \cdots, r_{n} \rightarrow 1} \frac{\log T_{f \circ g}\left(r_{1}, r_{2}, \cdots, r_{n}\right)}{\log T_{g}\left(r_{1}, r_{2}, \cdots, r_{n}\right)}=\infty .
$$

We omit the proof of Theorem 8 because it can be carried out in the line of Theorem 7 .

Remark. Theorem 8 is also valid with "limit superior" instead of "limit" if ${ }_{v_{n}} \lambda_{f \circ g}^{L^{*}}=\infty$ is replaced by ${ }_{v_{n}} \rho_{f \circ g}^{L^{*}}=\infty$ and the other conditions remaining the same.

In the line of Corollary 1, we may easily verify the following:

Corollary 2. Under the assumptions of Theorem 8 or Remark 2,

$$
\lim _{r_{1}, r_{2}, \cdots, r_{n} \rightarrow 1} \frac{\log T_{f \circ g}\left(r_{1}, r_{2}, \cdots, r_{n}\right)}{\log T_{g}\left(r_{1}, r_{2}, \cdots, r_{n}\right)}=\infty .
$$

The proof is omitted because it may be carried out in the line of Corollary 1 .

\section{Competing interests}

The authors declare that they have no competing interests. 


\section{Authors' contributions}

All authors have contributed to all parts of the article. All authors read and approved the final manuscript.

\section{References}

[1] A. K. Agarwal: On the properties of an entire function of two complex variables, Canadian J.Math. Vol. 20 (1968), pp.51-57.

[2] S. K. Datta, T. Biswas and P. Sen: Measure of growth properties of functions analytic in unit disc, International J. of Math. Sci. \& Engg. Appls. (IJMSEA), Vol. 8, No. IV (July, 2014), pp. 147-216.

[3] B. A. Fuks: Theory of analytic functions of several complex variables, Moscow, 1963.

[4] O. P. Juneja and G. P. Kapoor : Analytic functions-growth aspects, Pitman avanced publishing program, 1985.

[5] C. O. Kiselman: Plurisubharmonic functions and potential theory in several complex variables, a contribution to the book project, Development of Mathematics 1950-2000, edited by Jean Paul Pier.

[6] D. Somasundaram and R. Thamizharasi : A note on the entire functions of L-bounded index and L-type, Indian J. Pure Appl. Math., Vol.19 (March 1988), No. 3, pp. 284-293. 